\title{
Editorial
}

\section{Modeling Experimental Nonlinear Dynamics and Chaotic Scenarios}

\author{
José Roberto Castilho Piqueira, ${ }^{1}$ Elbert E. Neher Macau, ${ }^{2}$ \\ and Celso Grebogi ${ }^{3}$ \\ ${ }^{1}$ Telecommunication and Control Engineering Department, Polytechnic School, \\ The University of São Paulo, 05508-970 São Paulo, Brazil \\ ${ }^{2}$ Laboratório Associado de Matemática Aplicada e Computação (LAC), Instituto Nacional de Pesquisas \\ Espaciais (INPE), São Josè dos Campos, 12227-010 São Paulo, Brazil \\ ${ }^{3}$ Institute for Complex Systems and Mathematical Biology, King's College, University of Aberdeen, \\ Aberdeen AB24 3UE, UK
}

Correspondence should be addressed to José Roberto Castilho Piqueira, piqueira@lac.usp.br

Received 25 August 2009; Accepted 25 August 2009

Copyright (C) 2009 José Roberto Castilho Piqueira et al. This is an open access article distributed under the Creative Commons Attribution License, which permits unrestricted use, distribution, and reproduction in any medium, provided the original work is properly cited.

Thinking about nonlinearity in engineering areas, up to the 70s, was focused on intentionally built nonlinear parts in order to improve the operational characteristics of a device or system. Keying, saturation, hysteretic phenomena, and dead zones were added to existing devices increasing their behavior diversity and precision. In this context, an intrinsic nonlinearity was treated just as a linear approximation, around equilibrium points.

Approximation methods and Lyapunov stability theorems elegantly solved the dynamical behavior analysis problems and gave hints about how to synthesize systems with some desired properties. At that time, bifurcation and chaos were not present in engineers' daily life.

Chaotic dynamics was discovered at the end of the nineteenth century, by Henry Poincaré as a result from nonlinear phenomena subjected to qualitative changes in their behavior, provoked by bifurcations due to parameter variations. By studying the mathematical model of the circular restricted three-body problem, he was able to glimpse the chaotic motion that appears in complicated and apparently unpredictable trajectories that were close to periodic orbits, but spread fully in bounded regions of the phase space. Analyzing this motion, he concluded that "... it may happen that small differences in the initial conditions produce very great ones in the final phenomena. A small error in the former will produce an enormous error in the latter. Prediction becomes impossible, and we have the fortuitous phenomenon."

In the last couple of decades of the twentieth century, the profound impact of chaos in science, engineering, and medicine was acknowledged. Fundamental experiments performed 
in all areas of science allowed us to finally understand a realistic scenario in which the chaotic behavior not only occurs in Nature, but it is also a fundamental mechanism that Nature accounts for to mediate its phenomena. Thus, the chaotic behavior can be observed, for example, in the electrical activity from biological systems, in the transition of a fluid to turbulent motion, and in the motion of the moons of the giant planets.

Inspired on the rediscovering of the richness of nonlinear and chaotic phenomena, engineers started using analytical tools from "Qualitative Theory of Differential Equations," allowing more precise analysis and synthesis, in order to produce new vital products and services. Bifurcation Theory, Dynamical Systems, and Chaos started to be part of the mandatory set of tools for design engineers.

Consequently, an important interaction between Mathematics and Engineering started to gain importance and, in this scenario, the blending of three distinct methodological approaches, analytical, numerical, and experimental needs to be improved as follows.

(i) Numerical experiments that cleverly and precisely use tailored computer-based numerical simulations to give insights into problems that are analytically intractable so far.

(ii) Analytical methods to deal with nonlinear differential equations and to geometrically describe structures that arise as a consequences of the chaotic dynamics.

(iii) High precision experiments, which involve up-to-date measurement technology and procedures to capture the chaotic behavior in specific fields.

This special edition of the Mathematical Problems in Engineering aims to provide a picture of the importance of the Bifurcation Theory, relating it with nonlinear and chaotic dynamics for natural and engineered systems. Ideas of how this dynamics can be captured through precisely tailored real and numerical experiments and understanding by the combination of specific tools that associate dynamical system theory and geometric tools in a very clever, sophisticated, and at the same time simple and unique analytical environment are the subject of this issue, allowing new methods to design high precision devices and equipment.

Following these lines, this special issue contains 25 papers organized as follows. Concerning an overview of modeling nonlinear dynamics, there is one paper entitled "Modeling nonlinear dynamics and chaos: A review" by L. A. Aguirre and C. Letellier. Concerning an overview of the application of bifurcation theory to turbulence, there is one paper entitled "Flow around a slender circular cylinder: A case study on distributed Hopf bifurcation" by Aranha et al. Concerning applications of bifurcation theory to nonlinear mathematical and computation models, there are four papers entitled "Analysis of nonlinear dynamics for abrupt change of interphase structure in liquid-liquid mass transfer" by Zhang et al.; "Continuation of periodic solutions of dissipative and conservative systemsapplication to elastic pendulum" by Pavel Pokorny; "Dynamical analysis of an interleaved single inductor TITO switching regulator" by El Aroudi et al.; "Higher period stochastic bifurcation of nonlinear airfoil fluid-structure interaction" by J. A. S. Witteveen and H. Bijl. Concerning computational and numerical techniques of analysis of models, there are four papers entitled "The effect of spatial scale on predicting time series: A study on epidemiological system identification" by Monteiro et al.; "Macro- and micro-simulations for a sublimation growth of SiC single crystals" by J. Geiser and S. Irle; "Free vibration analysis of rectangular orthotropic membranes in large deflection" ZHENG et al.; "Modified Jacobian Newton iterative method: Theory and applications" by Juergen Geiser. Concerning 
data analysis, there are three papers entitled "The staircase structure of the southern Brazilian continental shelf" by M. Baptista and L. A. Conti; "Solitons, peakons, and periodic cuspons of a generalized Degasperis-Procesi equation" by J. Zhou and L. Tian; "Chaotic patterns in aeroelastic signals" by F. Marques and R. M. G. Vasconcellos. Concerning synchronization and control, there are eight papers named "Limit cycle prediction based on evolutionary multi objective formulation" by Katebi et al.; "Intermittent behavior and synchronization of two coupled noisy driven oscillators" by Santos et al.; "Chaos synchronization between two different fractional systems of Lorenz family" by Ahmed Ezzat Matouk; "Diffusive synchronization of hyperchaotic Lorenz systems" by Ruy Barboza; "Synchronization of discrete-time chaotic systems in bandlimited channels" by Eisencraft et al.; "Trajectory sensitivity method and master-slave synchronization to estimate parameters of nonlinear systems" by Cari et al.; "Adaptive step-size control in simulation of diffusive CVD processes" by J. Geiser and C. Fleck; "A new mechanical model for particle transport by surface waves and applications" by Ragulskis et al. Concerning trans-disciplinary applications, there are four papers entitled "Nonlinear dynamics and chaos in a fractional-order HIV model" by H. Ye and Y. Ding; "Chaotic Image encryption design using Tompkins-Paige algorithm" by S. E. Borujeni and M. Eshghi; "An LPV fractional model for canal control" by Martinez-González et al.; "Flux reflection model of the ferroresonant circuit" by Milicevic et al.

As guest editors for this special issue, we wish to thank all those who submitted manuscripts for consideration. We also would like to thank the many individuals who served as referees. We hope that these articles can motivate and foster further scientific works that will allow for a continuous and better understand of the role of the chaotic dynamics in our world.

José Roberto Castilho Piqueira

Elbert E. Neher Macau Celso Grebogi 


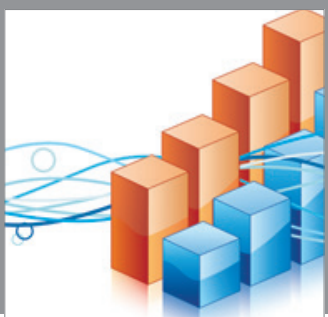

Advances in

Operations Research

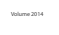

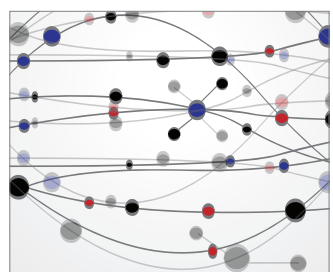

\section{The Scientific} World Journal
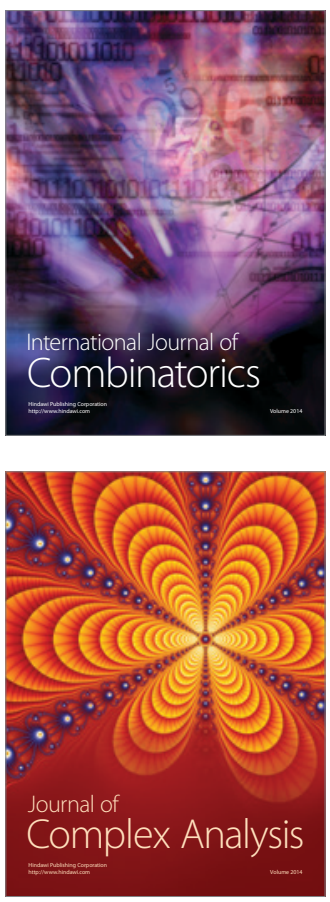

International Journal of

Mathematics and

Mathematical

Sciences
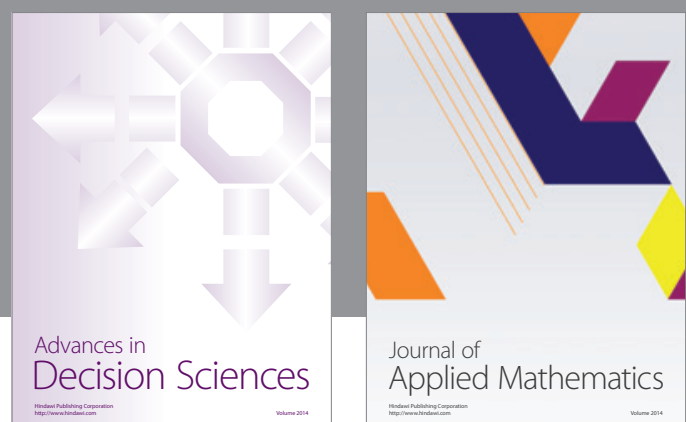

Journal of

Applied Mathematics
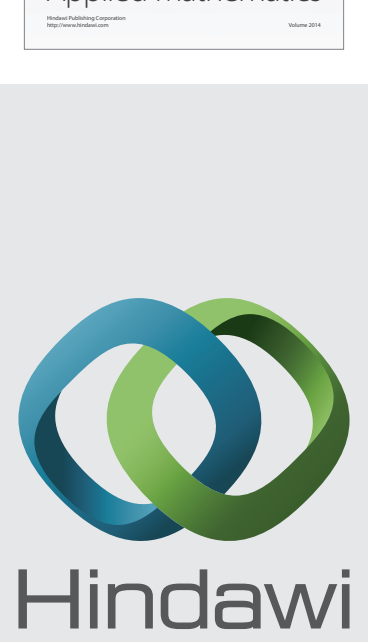

Submit your manuscripts at http://www.hindawi.com
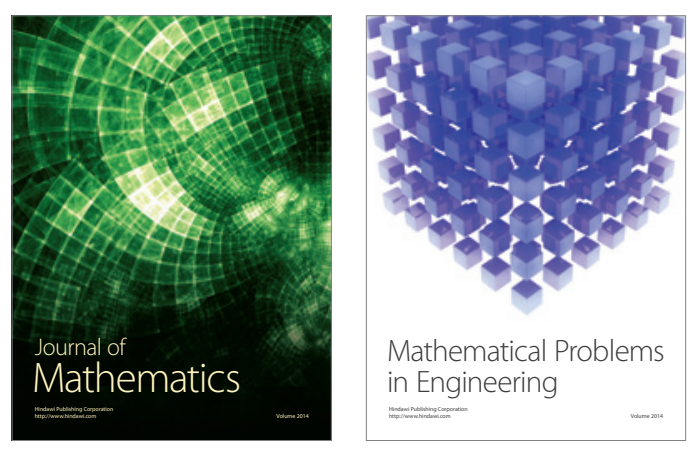

Mathematical Problems in Engineering
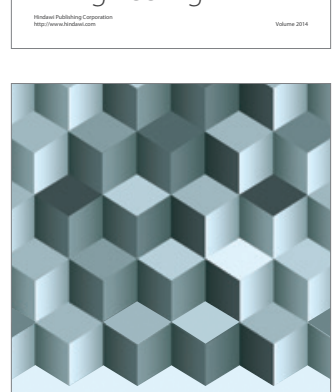

Journal of

Function Spaces
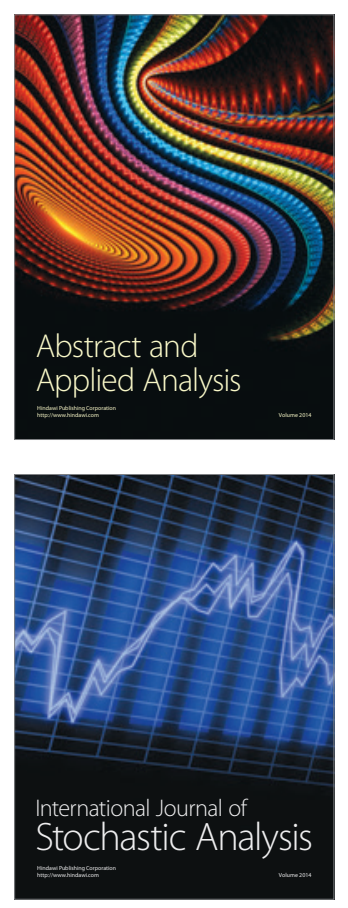

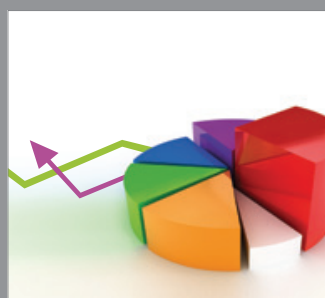

ournal of

Probability and Statistics

Promensencen
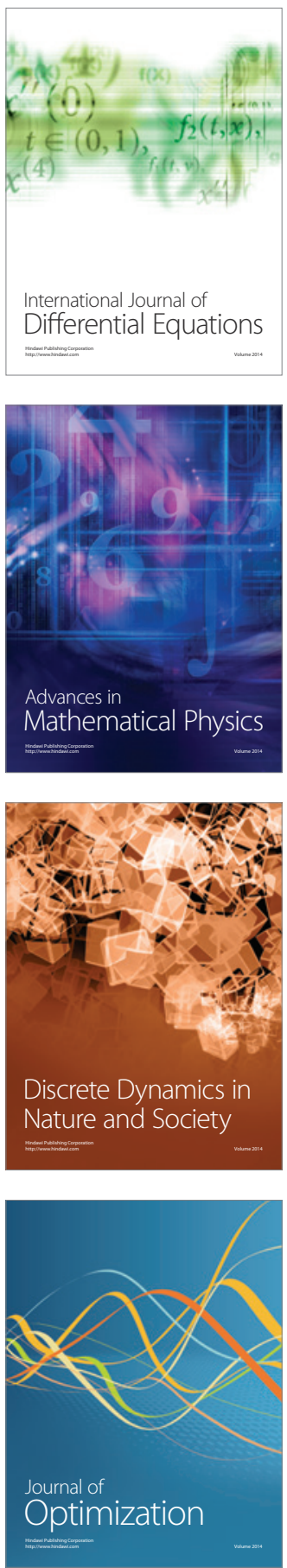